\title{
Fault Diagnostics of Wind Turbine Drive-Train using Multivariate Signal Processing
}

\author{
R. Uma Maheswari \\ Anna University, Assistant Professor (Senior Scale), Rajalakshmi Institute of Technology, Chennai-600124, India.
}

\section{R. Umamaheswari}

Velammal Engineering College, Chennai-600066, India.

\author{
(Received 5 June 2018; accepted 18 February 2019)
}

\begin{abstract}
The vibration measured from wind turbine drivetrain components is a mixture of multiple frequency modes. In practice, in wind turbine drivetrain condition monitoring systems, multiple accelerometer sensors are used to measure the vibration. Inter-channel common modes are not processed in the standard single-channel empirical mode decomposition (EMD) and it suffers from mode mixing and mode misalignment. Inter-channel correlation implies the causation of vibration mode shapes. Multivariate EMD (MEMD) possesses an enhanced spatial and spectral coherence. The mode alignment property of MEMD is used to process the inter-channel common modes, thus MEMD overcomes the limitation of mode misalignment in single-channel EMD. Still, MEMD exhibits a degree of mode mixing. White noise powers are added in separate channels to lessen the mode mixing. In this research, a novel multivariate signal processing technique, noise-assisted multivariate empirical mode signal decomposition (NA-MEMD) with a competent nonlinear Teager-Kaiser energy operator (NLTKEO), is proposed and tested for truthful extraction of instantaneous frequency and instantaneous amplitude features, and thereby ensures superior fault diagnosis performance. The dyadic filter bank structure of the proposed NA-MEMD decomposes the nonstationary vibrations effectively. The proposed method is used to predict the surface damage pattern embedded in multi-source vibrations at a low-speed planetary gear stage. The effectiveness of the proposed algorithm is tested with NREL GRC wind turbine condition monitoring benchmark datasets.
\end{abstract}

\section{INTRODUCTION}

The contribution of wind power in the renewable energy sector is growing exponentially. The cost of wind energy is proportional to the operation and maintenance (O\&M) cost of the wind farms. Unscheduled downtime of the turbines increases O\&M cost and also affects their reliability. Predictive condition monitoring is a promising maintenance strategy that reduces the maintenance costs and optimizes the availability of wind energy. The condition-based maintenance strategy is capable of predicting the system component failure at the incipient stages, thereby abrupt faults can be prevented. With the recent advent of sensor technology, the condition monitoring of wind forms shows excellent capabilities in measuring the rotating machine vibration with more precision. Vibration analysis is one of the condition-based monitoring techniques that has been practiced in the industry at large. Time domain and frequency domain analysis of measured vibration signals are exhausted since these traditional signal processing algorithms are appropriate only for the stationary operating conditions. Wind turbines are nonlinear systems operating at non-stationary operating conditions; thus, more sophisticated techniques are required for continuous monitoring and fault diagnosis. Li, et al. proposes fault diagnosis of planetary gears based on multiscale symbolic dynamic entropy. Bandwidth-based envelope interpolation is proposed for rolling bearing fault diagnostics. ${ }^{1-4}$ Various decomposition techniques like intrinsic scale decomposition and local scale decomposition have been studied in both $\mathrm{Yu}$ and Liu and Yu and Lv to extract the weak fault features from rolling bearings. 5,6 The local mean decomposition is a variant of EMD, which has been applied to decompose the vibration signal into the product of envelope and frequency modulated signal. From this product function, the instantaneous frequency was estimated to detect the gear crack fault frequencies. $^{7}$

To eliminate the mode mixing problem in EMD, ensemble empirical mode decomposition (EEMD) was used by the energy separation algorithm for fault diagnosis. ${ }^{8}$ Yu, et al. applied the EMD method to detect the faults of roller bearings. The wavelet-based de-noising was employed on a vibration signal envelope to extract fault patterns. ${ }^{9}$ Diagnosing bearings faults by using EMD with variants was studied in Yu, et al., and in Rai and Mohanty. ${ }^{10,11}$ The extracted energy of each intrinsic mode function (IMF) was used as a feature to train the ANN. ${ }^{10}$ A Hilbert transform and FFT were used to extract the envelope spectrum of computed IMF to diagnosis the bearing fault signatures. ${ }^{11}$ Yang, et al. proposed the condition monitoring system for wind turbines by applying EMD on the vibration signal. That amplitude modulation technique on resultant IMF was studied to correlate different types of faults. ${ }^{12}$

The major drawbacks in standard single-channel EMD are mode mixing and mode misalignment problems. Mode mixing means different oscillation modes are present in a single IMF and mode misalignment corresponds to the appearance of the same mode across different IMFs. ${ }^{13}$ In wind turbine vibration monitoring, the data are collected at multiple locations with multiple accelerometer sensors and the acquired signals are multivariate in nature. As in simple single-channel approaches, if these vibration signals sensed at different locations are analysed separately, the location information could 
be lost. Inter-channel correlation among vibration modes improves the decomposition efficacy by exploiting mode alignment property of dyadic filter structure in multivariate signal processing. Rehman, et al. proposed the multivariate EMD (MEMD) by projecting the input signal to multiple direction vectors in multi-dimensional space. Multivariate EMD extracted the common mode of oscillations within the multivariate data. ${ }^{14}$ The genesis of multivariant EMD is rooted in the work extending the standard EMD to a complex domain by estimating the complex-valued envelop with the help of spline interpolation. IMFs are extracted by using the rotation invariant property of the phase. ${ }^{15,16}$ Univariate EMD is extended into a bivariate time series by projecting the input signal into different directions in complex two-dimensional space and extracts the local mean from the envelops. ${ }^{17}$ The EMD is extended into a trivariate time series by projecting the input signal in multiple directions in three-dimensional space. ${ }^{18}$ Single-channel EMD and EEMD are extensively used as the fault diagnostic tools in rotating machinery condition monitoring. The multivariate EMD is used in bio signal analysis to detect anomalies. Only a very few works have studied the potential of multivariate EMD in the fault diagnostics of rotating machinery distributive surface fatigue detection. Bivariate EMD is employed to detect the incipient faults in wind turbine shafts. ${ }^{19}$ Full spectrum MEMD is proposed for rotating machinery condition monitoring. Impeller vane trailing edge damage detection is carried out by using MEMD.$^{20} \mathrm{In} \mathrm{Lv}$, et al., MEMD was applied to the roller bearing fault diagnostics. ${ }^{21}$ A non-local means algorithm was used as the pre-processing method to improve the sensitivity of the MEMD. The authors did not discuss the mode mixing problem of the MEMD. In previous works, the efficacy of the MEMD was analysed in rolling bearings, pumps, etc. The wind turbine transmission systems are complex in nature and the vibration signal measured at the drivetrain are a compound of the drivetrain components. In the standard fault diagnostic methods, the single-channel EMD with a Hilbert transform is employed to extract the features from raw vibration signals.

A nonlinear Teager-Kaiser energy operator is simple to implement and offers excellent temporal resolutions. ${ }^{22}$ In Alsonso, et al., a Teager-Kaiser energy operator was directly applied to the bearing raw vibration signal to extract the AM modulated features. ${ }^{23}$ In Zhao and $\mathrm{Li}$, wind turbine bearing fault diagnosis was carried out by a Teager-Kaiser energy operator applied to a sensitive intrinsic mode function derived from variational mode decomposition. ${ }^{24}$

Wind turbines operate under highly non-stationary operating conditions, so the mode mixing issue will affect the performance of the fault diagnostics. To mitigate the mode mixing problem in a multivariate approach, NA-MEMD is implemented. White Gaussian Noise (WGN) is added as the separate channels to enhance the extraction of local extremes. ${ }^{25}$ WGN is band-limited noise added as separate parallel channels to retain the dyadic filter bank structure.

In this work, a novel fault diagnostic tool based on NAMEMD-TKEO is proposed for wind turbine drivetrain fault diagnostics. Wind Turbine Gearbox Vibration Condition Monitoring Benchmarking Datasets, provided by NREL, are used to validate the proposed method. Planetary gear stage fault diagnosis is carried out. A visual inspection of instantaneous amplitude spectrum and instantaneous frequency demodulation indicates the failure modes such as polishing wear, se-
Table 1. Gear Details

\begin{tabular}{cccc}
\hline Gear Element & $\begin{array}{c}\text { Number of } \\
\text { Teeth }\end{array}$ & Mate Teeth & Gear Ratio \\
\hline Ring Gear & 99 & 39 & \\
Planet Gear & 39 & 99 & 5.71 \\
Sun Pinion & 21 & 39 & 3.57 \\
Intermediate & 82 & 23 & 4.0 \\
$\quad$ Gear & & 22 & \\
High-Speed & 88 & & \\
Gear & &
\end{tabular}

vere scuffing, and fretting corrosion. The key challenge is to extract the instantaneous features from the specific vibration measurement, which gives information about multiple components. The fault signatures are embedded within the information. A fruitful extraction depends on the accuracy of the intrinsic mode decomposition; hence, in this study, an attempt is made to use the potential of multivariate decomposition to extract the more meaningful intrinsic mode functions. The instantaneous feature profile changes rapidly with the changes in the signal. NLTKEO is more sensitive to the changes in the vibration signal. Through the development of the surface fatigue fault diagnostics framework, the authors contribute as follows:

1. Surface fatigue adhesive wear, such as scuffing and fretting corrosion, are distributive in nature. In this proposed framework, surface wear detection at the incipient stages is carried out by using the mode alignment property of the MEMD. To analyse the distributive faults, common scale oscillatory modes are to be synchronized in common scale alignment. The proposed method exploits the advantage of the MEMD dyadic filter banks structure's mode alignment property to decompose the distributive multi-component vibration time series.

2. Inter-channel correlation among multiple sensor fusion is adapted to decompose multimode components into monointrinsic modes in the distributive spectrum.

\section{MATERIALS AND METHODS}

\subsection{Vibration Data Set}

The vibration data used in this study were obtained from NREL Wind Turbine Gearbox Vibration Condition Monitoring Benchmarking Datasets. The GRC test wind turbine was a stall controlled, three-bladed upwind turbine with rated power $750 \mathrm{~kW}$. National instrument PXI-4472B was the high-speed data acquisition system. Vibration signals were measured at $40 \mathrm{kHz}$ per channel. Eight accelerometer sensors were located at various places in the wind turbine to measure the vibration data. The dataset consisted of ten one-minute segments of data measured at 50\% rated power and an 1800 RPM nominal high-speed shaft. The corresponding main shaft speed is 22.09 RPM. The experimental setup consisted of two gearboxes with an overall gear ratio of 1:81.49. The gearboxes consisted of one low-speed planetary stage and two parallel stages. Table reftab.1 lists the gear details used in low-speed planetary gear stage. 


\subsection{Methods}

\subsubsection{Noise-Assisted Multivariate Empirical Mode Decomposition}

Basically, multivariate EMD is a self-adaptive data-driven method. The standard EMD identifies the principal oscillating mode from the local mean of the upper and lower signal envelops and this local mean is subtracted iteratively from the signal until the stopping criteria are satisfied. The principal intrinsic oscillating mode was subtracted from the signal to form the residue and the procedure was repeated on the residue to obtain another IMF. In the multivariate signal, the local minima and maxima were not properly defined, hence the local mean could not be calculated. Rehman, et al. proposed that the signal of interest is projected along different directions in multi-dimensional space by a suitable set of direction vectors. ${ }^{14}$ The local mean was calculated by averaging the projections in multi-dimensional space. The choice of direction vectors depended on the scheme of uniform sampling along a hypersphere. Sampling based on low discrepancy Halton and Hammersley sequences derived from the quasi-Monte Carlo method was a more appropriate choice to generate a uniform point set on multidimensional sphere. ${ }^{14}$ Generation of Hammersley sequence was based on the Van der Corput sequence that was defined as:

$$
\Phi_{m}(j)=\frac{x_{0}}{m}+\frac{x_{1}}{m^{2}}+\frac{x_{2}}{m^{3}}+\cdots+\frac{x_{r}}{m^{r+1}},
$$

where $m_{0}, m_{1}, m_{2}, \ldots, m_{r}$ were the first $r$ prime numbers, $\Phi_{m}(j)$ for $j=0,1,2$, was Van Der Corput sequence, where $x$ was sampled value. ${ }^{26}$ The non-integer $j$ in the prime base $\mathrm{m}$ was defined as:

$$
j=x_{0}+x_{1} m+x_{2} m^{2}+\cdots+x_{r} m^{r} .
$$

Letting $n$ be the dimension of the space to be sampled and the $m_{1}, m_{2}, \ldots, m^{n-1}$ sequence of prime numbers, then $n$ dimensional Hammersley points were:

$$
\begin{gathered}
\left(\frac{m}{k}, \Phi_{m_{1}}(j), \Phi_{m_{2}}(j), \ldots, \Phi_{m_{n-1}}(j)\right), \\
\text { for } j=0,1,2, \ldots, k-1,
\end{gathered}
$$

where $k$ was the total number of Hamersley points when directional vectors were generated by mapping Hammersley points on a spherical surface. First, the Hammersley points are mapped in the cylindrical domain as follows:

$$
\begin{gathered}
\left(\frac{m}{k}, \Phi_{m}(j)\right) \rightarrow(\phi, t), \\
(\phi, t) \in[0,2 \pi] \times(1,-1) .
\end{gathered}
$$

Then, the unit cylinder was projected along the radius to map into the unit sphere:

$$
(\phi, t) \rightarrow\left(\sqrt{\left(1-t_{2}\right)} \cos \theta, \sqrt{\left(1-t_{2}\right)} \sin \theta, t\right)^{T} .
$$

The Hammersley sequence provided uniform distributed direction vectors. The maxima and minima of the projections along the direction vectors gave the multidimensional envelope. The multivariate signal mean was obtained by averaging the multiple envelopes. The stoppage criterion did not impose the equality of zero crossings and the extreme, as in the standard EMD.
Regardless of the advantages, MEMD still suffered from a degree of mode mixing when decomposing the non-stationary signals. To alleviate this, the uncorrelated WGN was added in the separate channels to align the IMF without mode mixing. With the added noise channels, the MEMD oriented the different IMF, in accordance with the dyadic filter bank structure. Each IMF represented a separate frequency sub-band, thus it eliminated the mode mixing. ${ }^{27}$

\section{Noise-Assisted Multivariate EMD}

1. Uncorrelated white noise of the same length as the input multivariate signal with different SNR are added in separate channels with the input signal.

2. Hammersley point sets are chosen and projected along directional vectors.

3. Maxima of the projections are determined.

4. Envelops are calculated by interpolating the extremes.

5. The mean of envelopes is calculated.

6. The residue is calculated by subtracting the mean from the signal. If the residue satisfies the stoppage criteria steps 2-6 is iteratively applied on the subsequent residues.

7. Discards the IMF channels corresponding to the added noise channels.

In NA-MEMD, added WGN reinforced the dyadic filter structure. So the alignment of the common scale oscillating modes of the different channels was synchronized. To avoid leakage from the noisy channels into the input signal channels, the number of noise channels and the amplitude of noise power had to be chosen carefully. In this paper, four channels of WGN with SNRs $0 \mathrm{~dB}, 1 \mathrm{~dB}, 3 \mathrm{~dB}, 5 \mathrm{~dB}$ were added.

Mode mixing affects the dyadic filter behaviour of the MEMD. To maintain the dyadic filter structure, the bandlimited WGN noises were added in parallel channels. Timelocalized mode mixing was estimated by wavelet coherence.

\subsection{Nonlinear Teager-Kaiser Energy Operator}

An NLTKEO was used to estimate the energy envelop of the mono-component signal. In discrete time, the nonlinear operator was defined by Maragos, et al. as: $:^{28}$

$$
\Psi_{d}[y(n)]=y^{2}(n)-y(n+1) y(n-1),
$$

where $\Psi$ was the Teager-Kaiser energy operator, and $y(n)$ was the signal. Discrete-time energy separation (DESA-2) is used to detect the envelope of the AM-FM demodulated signal. In this algorithm, 2 implied that the first order difference between the sample's times indices differs by 2 . The DESA- 2 used 3 sample symmetric differences to estimate the AM-FM demodulated envelop. The mathematical representation in Maragos, et al. defined time-varying AM-FM envelops as: ${ }^{29}$

$$
\frac{1}{2} \arccos \left[1-\frac{\Psi[y(n+1)-y(n-1)]}{2 \Psi[y(n)]}\right] \approx \Omega_{i}(n),
$$




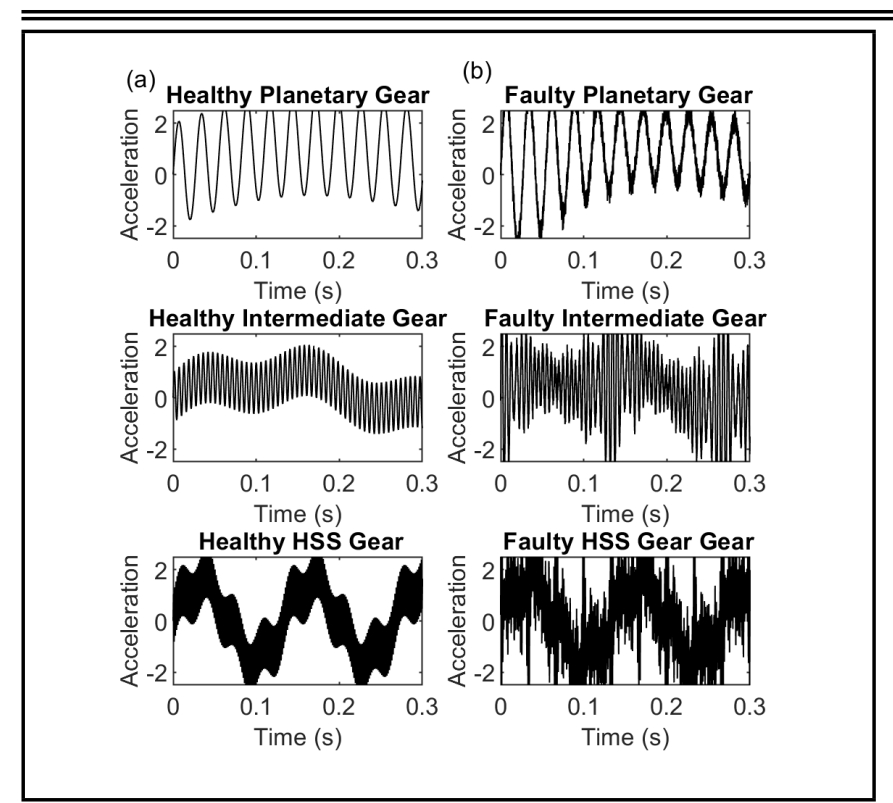

Figure 1. Simulated multi-component signal.

$$
\frac{2 \Psi[y(n)]}{\sqrt{\Psi[y(n+1)-y(n-1)]}} \approx|a(n)| .
$$

The first derivative of the signal of interest was approximated by symmetric difference to avoid half sample shifts in the estimated instantaneous frequency. ${ }^{29}$

\subsection{Proposed NA-MEMD-NLTKEO Method}

In a wind turbine drivetrain, the non-stationary vibrations are transmitted throughout the gear transmission systems. Considering the complexity involved in wind turbine drivetrain fault diagnosis, it was proposed to apply the multivariate timefrequency approach. Features can be extracted from the monocomponent stationary signal to detect and isolate the faults. A novel noise-assisted multivariate EMD decomposes the multicomponent into intrinsic mono components. The load/speed variations will cause the fundamental frequency and its harmonics smear into several frequency bins, which impose limitations in fault diagnostics. Usually, the vibration signal is sampled at a high sampling rate to avoid aliasing. Due to the large dynamic range of the measured vibration, the computational complexity is much higher. To increase the computational efficiency, the multi-channel data was time-segmented into 1 second blocks. The flow of the proposed NA-MEMDNLTKEO methodology is outlined as follows:

\section{NA-MEMD-NLTKEO Method}

1. Data acquisition system collects the vibration signal.

2. Multi-channel data are prepared from the various sensors.

3. Data segmented into 1 second blocks to reduce the computational overhead.

4. IMFs are extracted by applying the NA-MEMD as outlined in section 2. IMFs are representing the basic oscillating modes in the multivariate data.

5. IMFs are selected based on the energy content.

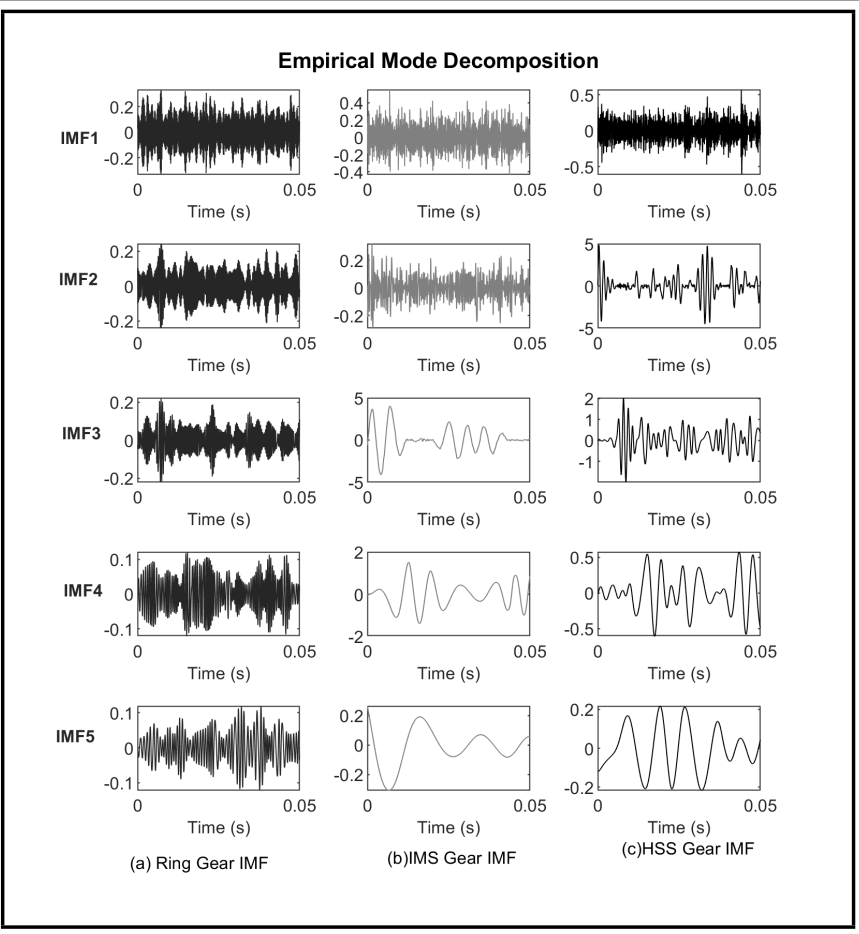

Figure 2. Multi-component decomposition using single-channel EMD.

6. Instantaneous amplitude and instantaneous frequency are estimated by the NLTKEO DESA 2 operator.

7. Envelop spectrum is determined from the estimated instantaneous amplitude and instantaneous frequency.

8. Amplitude-frequency modulation at Gear Meshing Frequency (GMF) and its harmonics indicate the failure modes.

\subsubsection{Simulation Results}

The efficacy of the proposed NA-MEMD-NLTKEO was tested on the synthesized multi-component AM-FM modulated signal.

$$
\begin{gathered}
x_{1}=A_{1} \sin \left(2 \pi f_{1} t\right)+A_{2} \sin \left(2 \pi f_{2} t\right)+A_{3} \sin \left(2 \pi f_{3} t\right) \\
x_{2}=A_{3} \cos \left(2 \pi f_{4} t\right)+A_{2} \sin \left(-2 \pi f_{6} t\right)+A_{1} \cos \left(2 \pi f_{5} t\right. \\
x_{3}=A_{1} \cos \left(2 \pi f_{7} t\right)+A_{3} \cos \left(2 \pi f_{9} t\right)+A_{2} \cos \left(-2 \pi f_{8} t\right.
\end{gathered}
$$

To simulate the pinion and gear vibration waveforms, the following gear mesh frequencies were considered. $G_{m e s h 1}$ was $36.5 \mathrm{~Hz}$ at the planetary stage, $G_{m e s h 2}$ was $172.5 \mathrm{~Hz}$ at intermediate speed stage, and $G_{m e s h} 3$ was $660 \mathrm{~Hz}$ at a highspeed stage. Main shaft frequency was $0.37 \mathrm{~Hz}$, planet shaft frequency was $1.30 \mathrm{~Hz}$, Sunshaft frequency was $2.10 \mathrm{~Hz}$, intermediate speed shaft was $7.50 \mathrm{~Hz}$, and the generator shaft was $30 \mathrm{~Hz}$. Figure 1 shows the simulated gear vibrations at healthy and faulty conditions.

Gear vibration waveforms are multicomponent signals, and to extract the useful features, the mono components must be decomposed. A standard single-channel EMD was applied to each signal separately, as shown in Fig. 2. The number of intrinsic mode functions extracted from each signal differed 


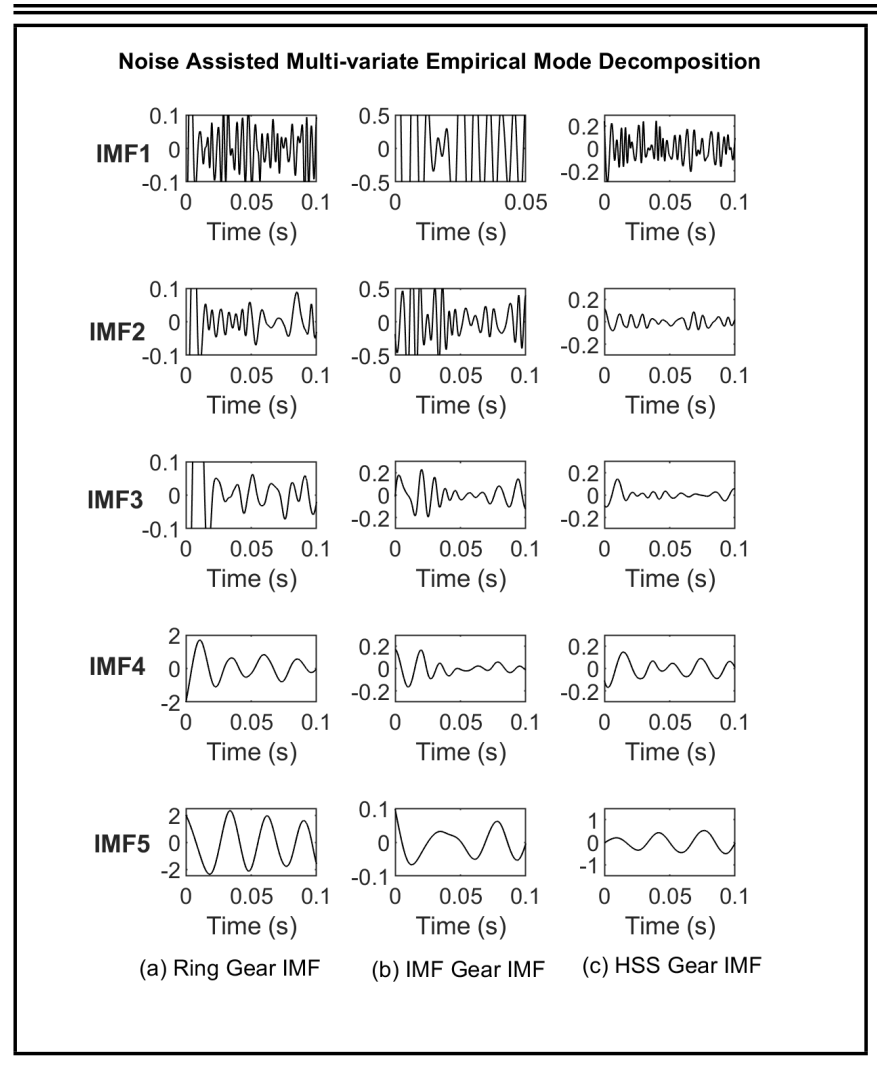

Figure 3. Multi-component decomposition using noise assisted multivariate EMD.

and the common modes did not extract properly. Figure 3 exhibits an IMF from NA-MEMD. The amplitude modulations were clearly indicated in the NA-MEMD decompositions and NA-MEMD prevented direct noise leakage into inter-channel decompositions. WGN channels separated the random component and a deterministic component with wider bandwidth; thus, it eliminated mode mixing.

\section{RESULTS AND DISCUSSION}

\subsection{Experimental Results and Discussion}

Vibrations measured from five accelerometer pickups in gear casing were used to formulate 5 channel multivariate data. Three noise channels with $3 \mathrm{~dB}, 15 \mathrm{~dB}$, and $20 \mathrm{~dB}$ were added in separate channels. NA-MEMD decomposed this octet-variate signal into $12 \mathrm{IMFs} / \mathrm{channels} \mathrm{(10} \mathrm{IMFs} \mathrm{are} \mathrm{shown} \mathrm{in} \mathrm{Fig.} 4$. A lower index IMF retained the high-frequency modes and a higher index IMF had the lowest frequency oscillating modes. Multi-components in multivariate data were decomposed into amplitude and frequency modulated mono components. The extracted number of IMFs and its order was the same for all five signals.

In the multivariate vibration signal, the quasi-dyadic subbands were more relevant, so that the added noise in the separate channels in the proposed method aligned the oscillating modes from the various channels. Mode mixing was evaluated by the time-varying correlation. Cross-correlation between the IMF was calculated to measure the similarity degree. In Fig. 5 a, it is observed that the IMFs extracted by NA-MEMD were less correlated and the wider peak in Fig. 5 b shows that the IMFs extracted by MEMD were highly correlated. Cross-correlation is effective only for stationary pro-
NA-MEMD Intrinsic Mode Decompostion

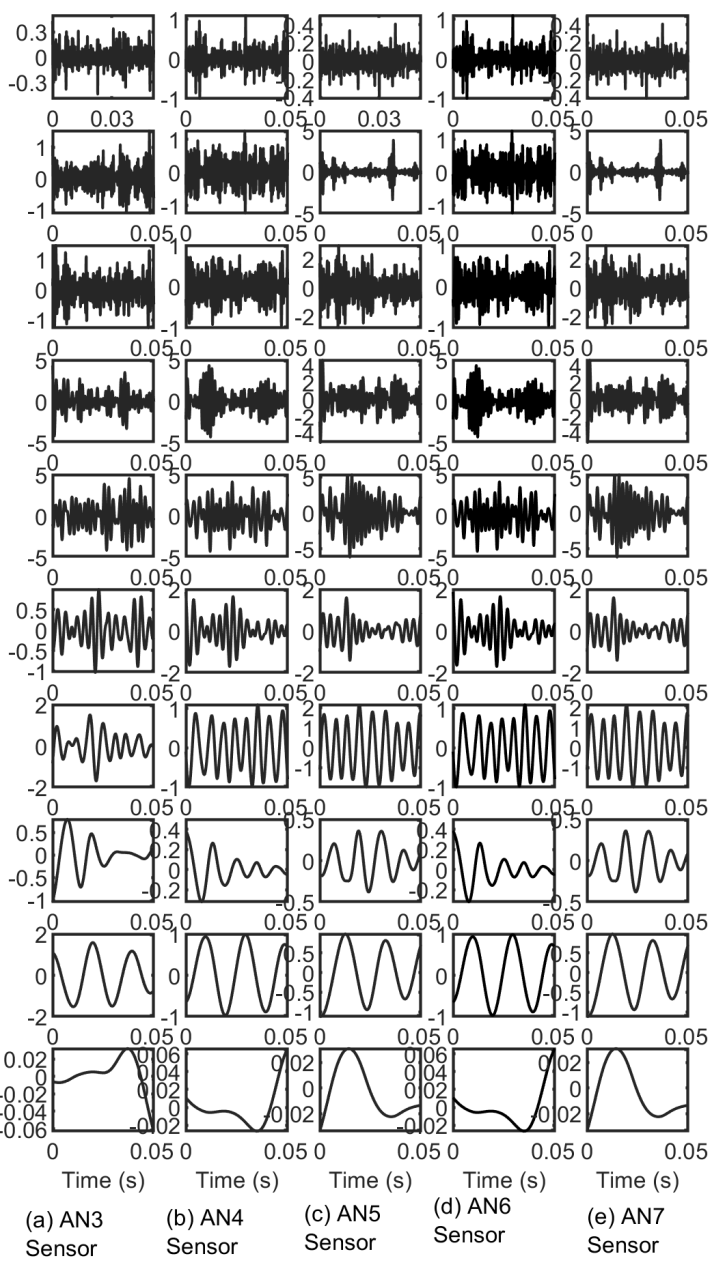

Figure 4. NA-MEMD decomposition of NREL condition monitoring bench marking dataset.

cesses to estimate time-varying correlation; wavelet coherence was adapted to analyse local common oscillation modes in the time-frequency plane. In Fig. 8, it is observed, that in the proposed NA-MEMD technique, the sub-band local oscillations were limited to $0.041 \mathrm{~Hz}$. In Fig. 7, MEMD spectral coherence shows significant oscillations, thus it suffered from severe mode mixing. The sets $0.03 \mathrm{~Hz}, 0.06 \mathrm{~Hz}, 0.125 \mathrm{~Hz}$ covary in IMF1 and IMF2. It is observed that, in Fig. 6, EMD spectral coherence indicates that $0.03 \mathrm{~Hz}$ and $0.125 \mathrm{~Hz}$ coexisted. The arrow indicates the phase lag between the IMFs. The cone of influence is represented by the edge effects at different frequency scales. It was observed that the relative phase lag oriented as 1/2 cycles in MEMD. It is observed in Fig. 5 b and in the EMD method, the $0.125 \mathrm{~Hz}$ oscillatory mode had a phase lag as $3 / 2$ cycles overlapped with a cone of influence. The IMFs instantaneous frequency and amplitude were estimated by using NLTKEO. NLTKEO is computationally simple and its temporal localization is much better. The amplitude envelope and the frequency envelope were determined from the instantaneous values. Table 2 lists the characteristic frequency of NREL turbine gears. 


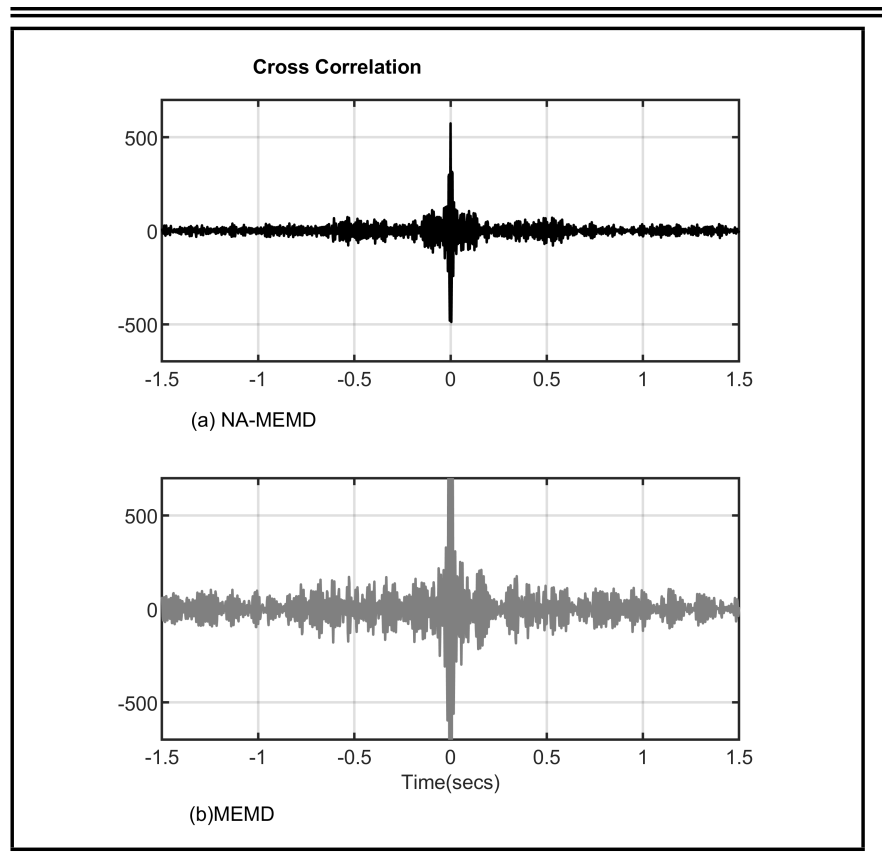

Figure 5. Cross correlation (a) NA-MEMD (b) MEMD.

Table 2. Planetary Stage Gear Characteristic Frequencies

\begin{tabular}{ccc}
\hline $\begin{array}{c}\text { Characteristic } \\
\text { Frequencies }\end{array}$ & Component & $\begin{array}{c}\text { Frequency } \\
(\mathrm{Hz})\end{array}$ \\
\hline Shaft Frequencies & MainShaft/Carrier & 0.37 \\
& Planets & 1.30 \\
Sun Shaft & 2.1 \\
Gear Mesh & Planet-Ring & 36.63 \\
Frequencies & Planet-Sun & 36.63 \\
& Sun-Intermediate & 172.5 \\
Assembly Phase & Planetary Stage & \\
Frequency & No. of Assembly & 12.21 \\
& Phase=3 & \\
Local Defect & Ring Gear & 1.10 \\
Frequency & Sun gear & 5.19 \\
& Planetary Gear & 2.60 \\
& Sun Gear & 1.73 \\
Distributed Fault & Ring Gear & 0.37 \\
Frequency & Planetary Gear & 0.93 \\
& & \\
\hline
\end{tabular}

\subsubsection{Planetary Gear Stage Fault Detection}

AN3 and AN4 sensor data were used to predict fault signatures that occurred at the planetary gear mesh stage. Figure 9 shows the AN3 sensor's dominant IMF envelope and power spectrum. It was observed that IMF2 and IMF3 were highly modulated in the frequency band [0.1-4.5] Hz. Figure 10 illustrates the IMFs envelope and its spectrum. The sideband energy levels were predominant in this frequency sub-band. Sideband energy distributed along the sub band indicated the surface damage. In Fig. 9, it is observed that order spectrum of IMF2 had peaks from 3.2 to 5.3 orders, which indicated the planetary gears were worn out. Ground truth in the NREL vibration dataset confirmed that the Annulus ring gear had scuffing patches. From each IMF order spectrum, the highest two peaks were extracted, as listed in table 3 . The order spectrum and power spectrum at their healthy states are illustrated in

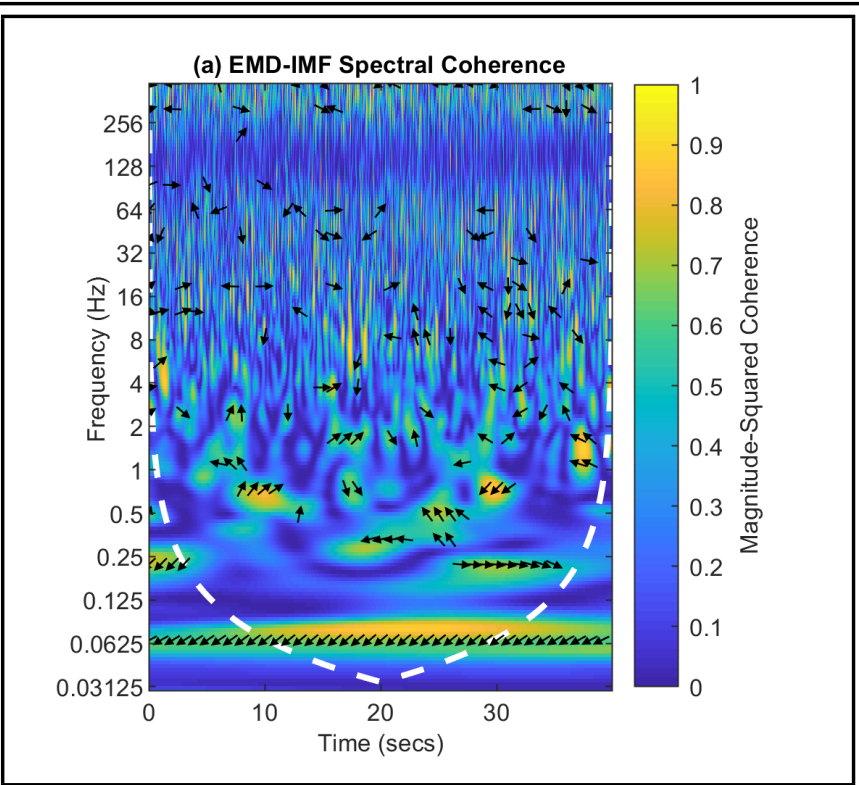

Figure 6. Spectral coherence of EMD-IMF coherence.

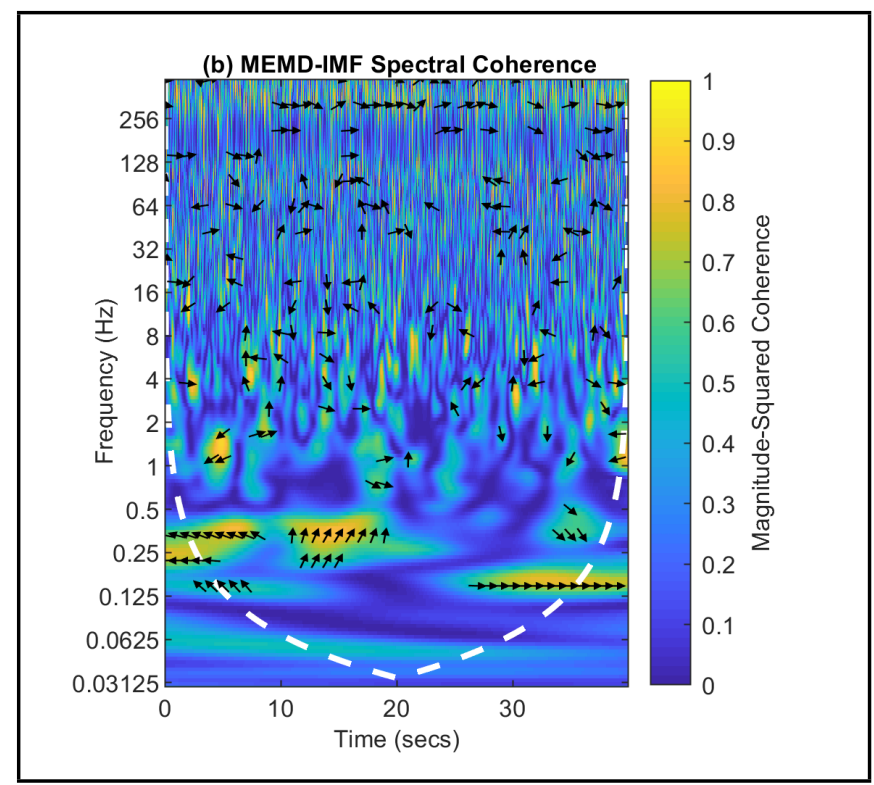

Figure 7. Spectral coherence of MEMD-IMF coherence.

Table 3. Peak Orders in Dominant IMFs

\begin{tabular}{cccc}
\hline Peak Orders & IMF 2 & IMF 3 & IMF 4 \\
\hline Faulty Condition & $4.64,3.98$ & $2.08,2.06$ & $1.65,1.24$ \\
Healthy Condition & $5.19,5.22$ & $2.90,2.75$ & $1.22,1.24$ \\
\hline
\end{tabular}

Fig. 10. Various speed turbines' planetary gear stage was subjected to multiple RPM under non-stationary operating conditions; order tracking was very effective in analysing the fault pattern.

\subsubsection{Performance Evaluation: Qualitative Analysis}

MEMD was an adaptive data-driven signal decomposition approach that decomposed the multi-components based on frequency scales, but the wavelets were a projection-based signal decomposition approach. In wavelet decomposition, prior knowledge is required to set the decomposition levels. Hence, wavelet-based decomposition is well suited for stationary processes. Wind turbines are operated at non-stationary condi- 


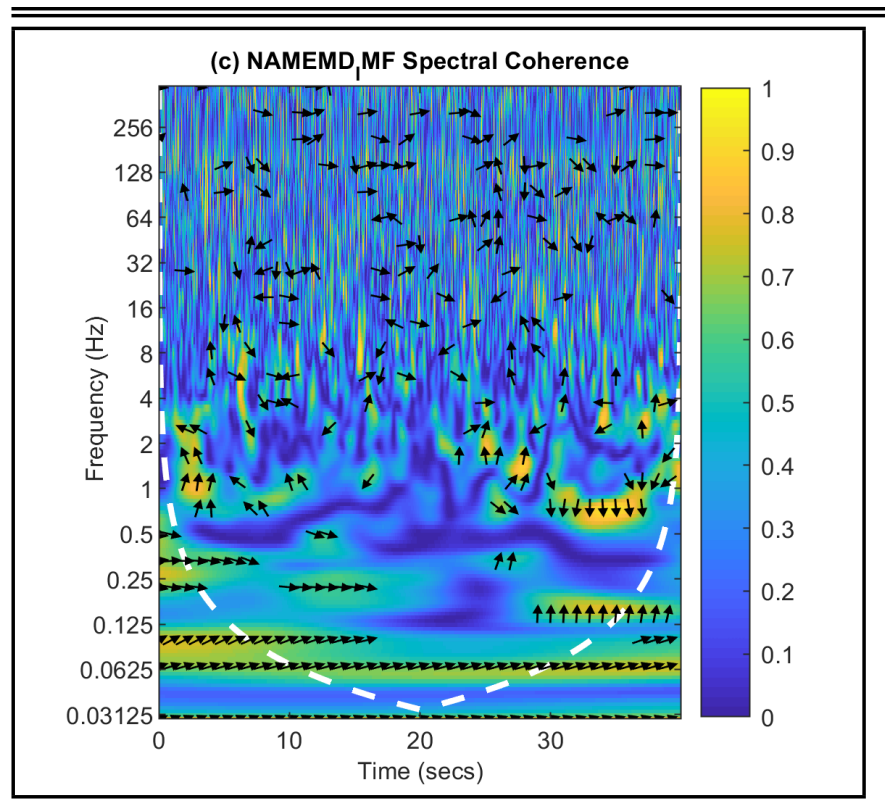

Figure 8. Spectral coherence of NAMEMD IMF.

tions, thus to set the decomposition levels prior will affect the decomposition performance. Furthermore, in wavelet decomposition, the oscillating modes were in variable lengths and were decomposed into smaller bandwidths, as shown in Figs. 11 and 12.

\subsubsection{Performance Evaluation: Qualitative Measure}

The performance of MEMD in decomposition was compared with wavelet decomposition by using quantitative analysis. The mean square error (MSE) was based on the Hurst exponent, signal to noise and distortion ratio (SINAD), total harmonic distortion (THD), and spectral entropy (SENT) were considered for validating. The Hurst exponent is the measure of the fractal dimension of a time series. In the present study intrinsic mode functions, scales were important to recognize the fault signatures. MSE was calculated with $H$ as 0.5 . Quantitative results of MEMD decomposition and wavelet decomposition is presented in Table 4. The MEMD outperformed wavelet decomposition.

\section{CONCLUSIONS}

Noise-assisted MEMD with a Teager-Kaiser nonlinear energy operator-based fault signature analysis is proposed for effective wind turbine drivetrain fault diagnosis. Hereby, using noise-assisted MEMD, the multicomponent vibration signal is first decomposed into mono-component modes and then a NLTKEO is applied to the resultant IMF signal components. The performance and effectiveness of the proposed NAMEMD-NLTKEO approach are investigated practically in real time with NREL Wind Turbine Gearbox Vibration Condition Monitoring Benchmarking Datasets. The observations and results of the combined signal processing strategy brought out promising results on the experimental dataset and complete analysis shows that the proposed approach is able to detect drivetrain faults; the residual signal obtained during signal decomposition for the experimentally measured nonlinear vibration signals highlights the fault condition more clearly when compared to an evaluated residual signal with simple HHT. The dominant frequencies in the IMF envelop spectrum can
Planetary Gear Vibration at Faulty Conditions
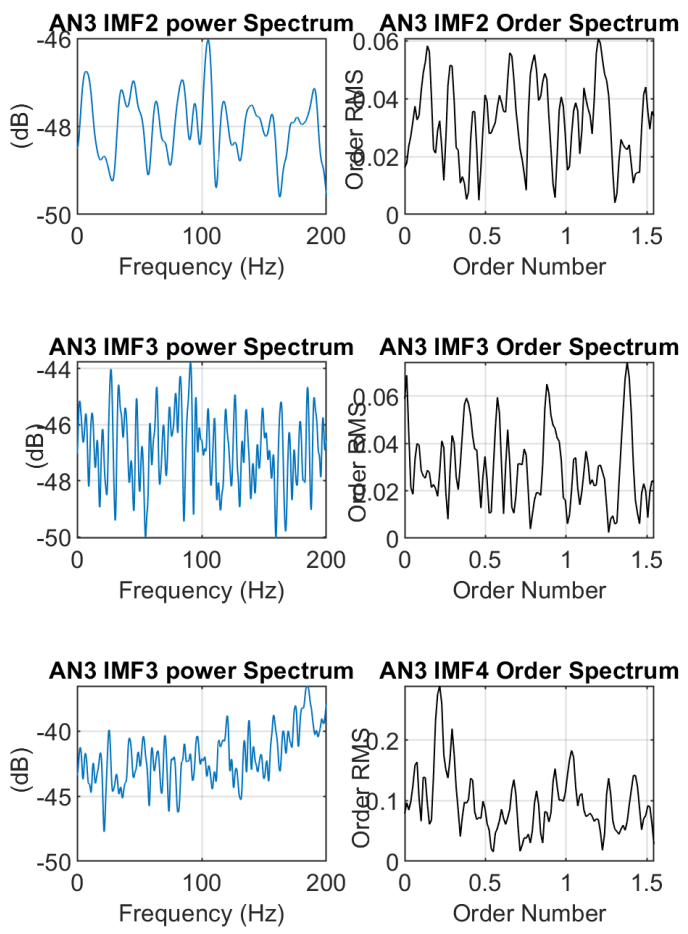

Figure 9. Planetary gear vibration power spectrum and order spectrum at fault conditions.

be related to the fault signature as sensitive and shows a significant increase in its value in the presence of gear tooth faults in the planetary stage. Thus, the proposed method has the potential to assess the degradation level of the gear mesh stiffness and also can be used to identify the fault location. Hence, the presented diagnosis approach in this paper is useful for developing an automatic diagnostic process in condition monitoring of offshore windmills.

\section{ACKNOWLEDGEMENTS}

We extend our gratitude to NREL for providing the Wind Turbine Gearbox Vibration Condition Monitoring Benchmarking Datasets.

\section{REFERENCES}

1 Li, Y., Xu, M., Wei, Y., and Huang, W. Rotating machine fault diagnosis based on intrinsic characteristicscale decomposition, MAMT, 94, 9-27, (2015). https://dx.doi.org/10.1016/j.mechmachtheory.2015.08.001

2 Li, Y., Li, G., Wei, Y., Liu, B., and Liang, X. Health condition identification of planetary gearboxes based on variational mode decomposition and generalized composite multi-scale symbolic dynamic entropy, ISA Trans., 81, 329-341, (2018). https://dx.doi.org/10.1016/j.isatra.2018.06.001

3 Li, Y., Feng, K., Liang, X., and Zuo, M. J. A fault diagnosis method for planetary gearboxes under non-stationary working conditions using improved Vold-Kalman filter and 
Table 4. Peak Orders in Dominant IMFs

\begin{tabular}{lcccccccc}
\hline Method & \multicolumn{3}{c}{ MEMD Decomposition } & \multicolumn{3}{c}{ Wavelet Decomposition } \\
\hline IMF & MSE & SINAD & THD & SENT & MSE & SINAD & THD & SENT \\
\hline IMF1 & 0.2752 & -17.94 & -28.54 & 0.8641 & 0.2979 & 0.88 & -6.35 & 0.9820 \\
IMF2 & 0.1835 & -17.24 & -12.27 & 0.7994 & 0.3039 & 8.54 & -Inf & 0.9807 \\
IMF3 & 0.0024 & -5.78 & -35.82 & 0.6717 & 0.3280 & 0.56 & -Inf & 0.9793 \\
IMF4 & 0.0816 & -3.20 & -46.84 & 0.6076 & 0.2914 & 7.35 & -Inf & 0.8652 \\
IMF5 & 0.1743 & -8.77 & -39.68 & 0.5485 & 0.1619 & -4.65 & -Inf & 0.9446 \\
IMF6 & 0.2250 & -0.12 & -33.11 & 0.5120 & 0.1896 & -4.19 & -9.51 & 0.9315 \\
IMF7 & 0.2418 & -2.43 & -10.11 & 0.4975 & 0.3380 & -1.24 & -25.69 & 0.8746 \\
IMF8 & 0.2475 & 6.11 & -56.06 & 0.4606 & 0.2335 & -3.12 & - Inf & 0.8594 \\
IMF9 & 0.2493 & -3.52 & -22.79 & 0.4108 & 0.2687 & -7.9 & -13.74 & 0.9484 \\
IMF10 & 0.2497 & 2.11 & -27.70 & 0.3951 & 0.1756 & -13.09 & -4.93 & 0.9552 \\
\hline
\end{tabular}

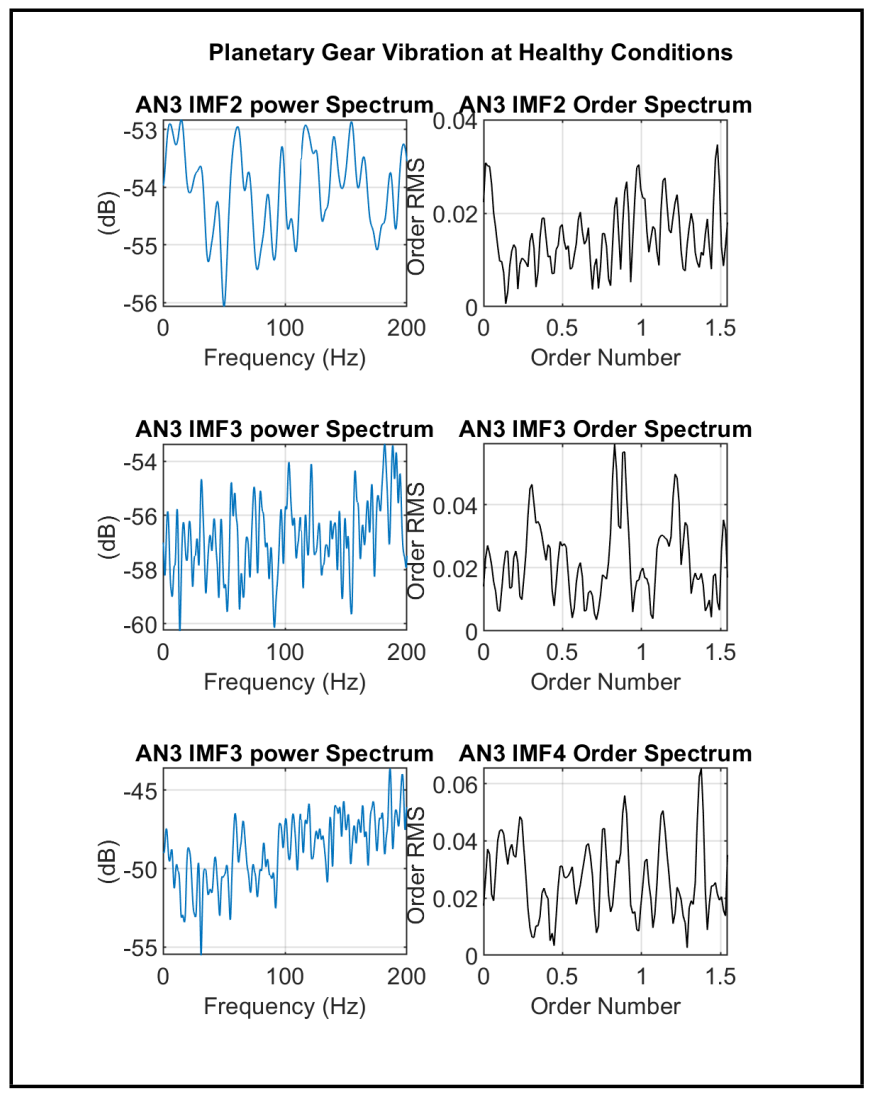

Figure 10. Planetary gear vibration power spectrum and order spectrum at healthy condition.

multi-scale sample entropy, J. Sound Vib., 439, 271-286, (2019). https://dx.doi.org/10.1016/j.jsv.2018.09.054

${ }^{4}$ Li, Y., Xu, M., Liang, X., and Huang, W. Application of bandwidth EMD and adaptive multiscale morphology analysis for incipient fault diagnosis of rolling bearings, IEEE Trans. Ind. Electron., 64 (8), 6506-6517, (2017). https://dx.doi.org/10.1109/TIE.2017.2650873

5 Yu, J. and Liu, H. Sparse coding shrinkage in intrinsic timescale decomposition for weak fault feature extraction of bearings, IEEE Trans. Instrum. Meas., 67 (7), 1579-1592, (2018). https://dx.doi.org/10.1109/TIM.2018.2801040

$6 \mathrm{Yu}$, J. and $\mathrm{Lv}, \mathrm{J}$. Weak fault feature extraction of rolling bearings using local mean decompositionbased multilayer hybrid denoising, IEEE Trans. Instrum. Meas., $66 \quad$ (12), 3148-3159, (2017). https://dx.doi.org/10.1109/TIM.2017.2751878

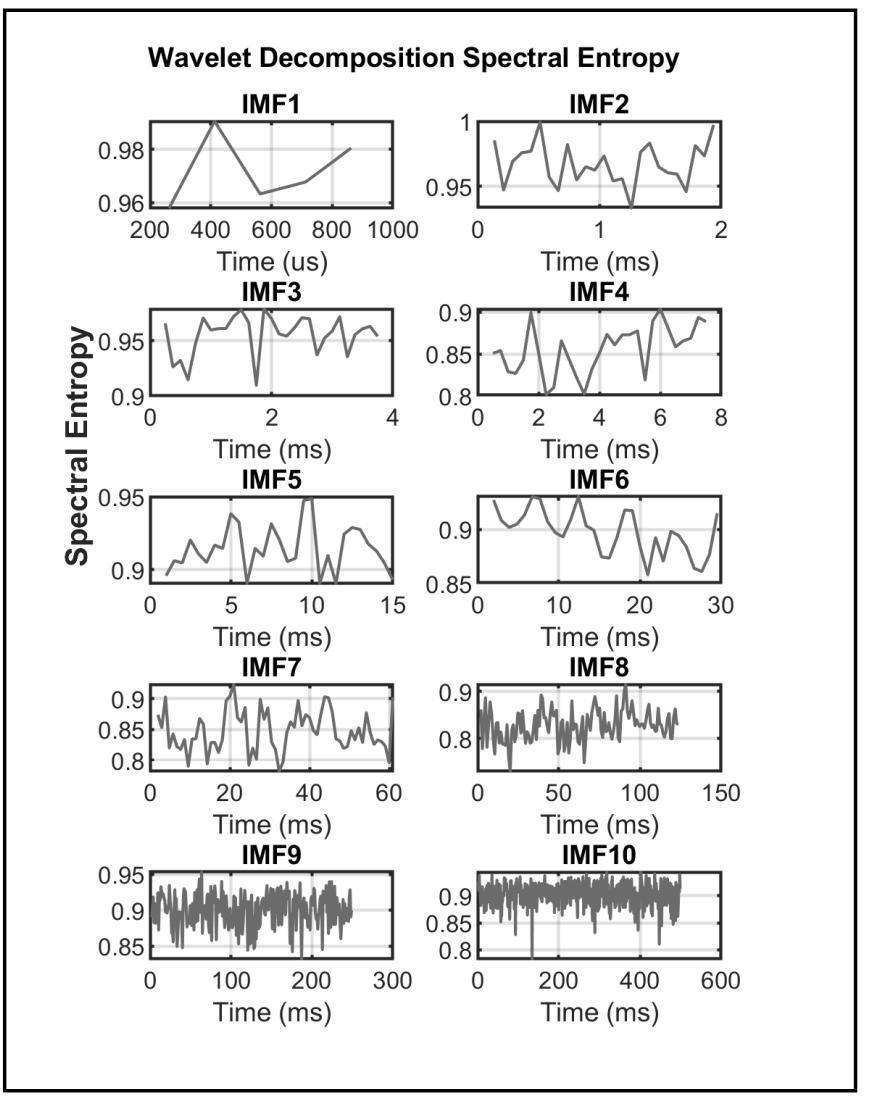

Figure 11. Spectral entropy of wavelet decomposed intrinsic mode functions.

7 Liu, W. Y., Zhang, W. H., Han, J. G., and Wang, G. F. A new wind turbine fault diagnosis method based on the local mean decomposition, Renew. Energy, 48, 411-415, (2012). https://dx.doi.org/10.1016/j.renene.2012.05.018

8 Feng, Z., Liang, M., Zhang, Y., and Hou, S. Fault diagnosis for wind turbine planetary gearboxes via demodulation analysis based on ensemble empirical mode decomposition and energy separation, Renew. Energy, 47, 112-126, (2012). https://dx.doi.org/10.1016/j.renene.2012.04.019

9 Yu, D., Cheng, J., and Yang, Y. Application of EMD method and Hilbert spectrum to the fault diagnosis of roller bearings, Mech. Syst. Signal Process., 19 (2), 259-270, (2005). https://dx.doi.org/10.1016/S0888-3270(03)000992

$10 \mathrm{Yu}, \mathrm{Y}$., YuDejie, and Junsheng, C. A roller bearing fault diagnosis method based on EMD energy entropy 


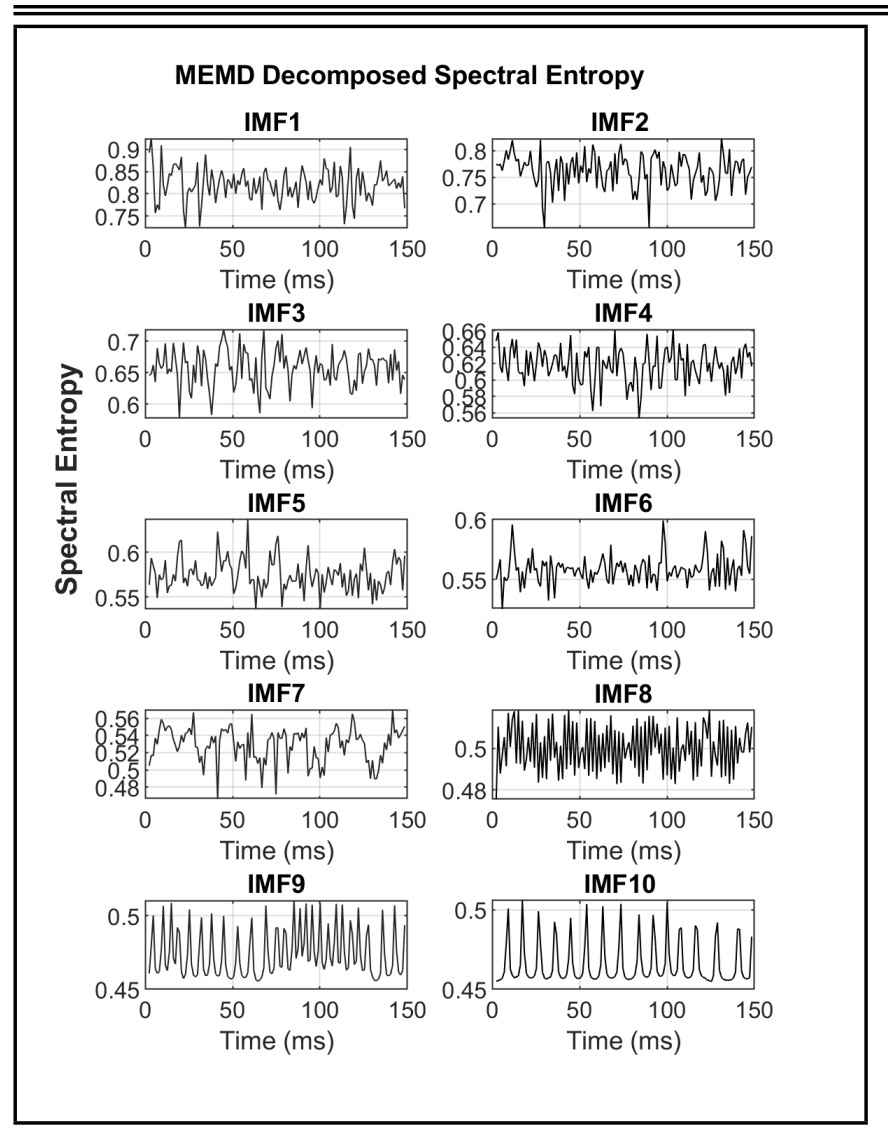

Figure 12. Spectral entropy of NA-MEMD decomposed intrinsic mode functions.

and ANN, J. Sound Vib., 294 (1-2), 269-277, (2006). https://dx.doi.org/10.1016/j.jsv.2005.11.002

11 Rai, V. K. and Mohanty, A. R. Bearing fault diagnosis using FFT of intrinsic mode functions in Hilbert-Huang transform, Mech. Syst. Signal Process., 21 (6), 2607-2615, (2007). https://dx.doi.org/10.1016/j.ymssp.2006.12.004

12 Yang, W., Tavner, P. J., and Crabtree, C. J. An intelligent approach to the condition monitoring of large scale wind turbines, European Wind Energy Conferene, Marseille, France, (2008).

${ }^{13}$ Looney, D. and Mandic, D. P. Multiscale image fusion using complex extensions of EMD, IEEE Trans. Signal Process., 57 (4), 1626-1630, (2009). https://dx.doi.org/10.1109/TSP.2008.2011836

14 Rehman, N. and Mandic, D. P. Multivariate empirical mode decomposition, Proc. R. Soc. A Math. Phys. Eng. Sci., 466, 1291-1302, (2009). https://dx.doi.org/10.1098/rspa.2009.0502

15 Altaf, M., U. Bin, Gautama, T., Tanaka, T., and Mandic, D. P. Rotation invariant complex empirical mode decomposition, IEEE Int. Conf. Acoust. Speech Signal Process., Honolulu, Hawaii, (2007).

16 Tanaka, T. and Mandic, D. P. Complex empirical mode decomposition, IEEE Signal Process. Lett., 14 (2), 101-104, (2007). https://dx.doi.org/10.1109/LSP.2006.882107

17 Rilling, G., Flandrin, P., Goncalves, P., and Lilly, J. M. Bivariate empirical mode decomposition, Sig- nal Process. Lett. IEEE, 14 (12), 936-939, (2007). https://dx.doi.org/10.1109/LSP.2007.904710

18 Rehman, N., Member, S., Mandic, D. P., and Member, S. Empirical mode decomposition for trivariate signals, IEEE Transcations on Signal Processing, 58 (3), 1059-1068, (2010). https://dx.doi.org/10.1109/TSP.2009.2033730

19 Yang, W., Court, R., Tavner, P. J., and Crabtree, C. J. Bivariate empirical mode decomposition and its contribution to wind turbine condition monitoring, J. Sound Vib., 330 (15), 3766-3782, (2011). https://dx.doi.org/10.1016/j.jsv.2011.02.027

${ }^{20}$ Zhao, X., Patel, T. H., and Zuo, M. J. Multivariate EMD and full spectrum based condition monitoring for rotating machinery, Mech. Syst. Signal Process., 27 (1), 712-728, (2012). https://dx.doi.org/10.1016/j.ymssp.2011.08.001

${ }^{21}$ Lv, Y., Yuan, R., and Song, G. Multivariate empirical mode decomposition and its application to fault diagnosis of rolling bearing, Mech. Syst. Signal Process., 81, 219-234, (2016). https://dx.doi.org/10.1016/j.ymssp.2016.03.010

22 Antoniadou, I., Manson, G., Dervilis, N., Barszcz, T., Staszewski, W. J., and Worden, K. Use of the TeagerKaiser energy operator for condition monitoring of a wind turbine gearbox, Proc. ISMA2012-USD2012, Leuven, Belgium, (2012).

23 Alonso, B., Ferrer, M. A., Travieso, C. M., and Henrı, P. Application of the Teager-Kaiser energy operator in bearing fault diagnosis, ISA Trans., 52, 278-284, (2013). https://dx.doi.org/10.1016/j.isatra.2012.12.006

${ }^{24}$ Zhao, H. and Li, L. Fault diagnosis of wind turbine bearing based on variational mode decomposition and Teager energy operator, IET Renew. Power Gener., 1-8, (2016). https://dx.doi.org/10.1049/iet-rpg.2016.0070

${ }^{25}$ Ur Rehman, N. and Mandic, D. P. Filter bank property of multivariate empirical mode decomposition, IEEE Trans. Signal Process., 59 (5), 2421-2426, (2011). https://dx.doi.org/10.1109/TSP.2011.2106779

26 Wong, T. T., Luk, W. S., and Heng, P. A. Sampling with Hammersley and Halton Points, J. Graph. Tools, 2 (2), 9-24, (1997). https://dx.doi.org/10.1080/10867651.1997.10487471

27 Ur Rehman, N., Park, C., Huang, N. E., and Mandic, D. P. Emd Via Memd: Multivariate noise-aided computation of standard emd, Adv. Adapt. Data Anal., 5 (2), 1350007, (2013). https://dx.doi.org/10.1142/S1793536913500076

${ }^{28}$ Maragos, P., Quatieri, T. F., and Kaiser, J. F. On amplitude and frequency demodulation using energy operators, IEEE Trans. Signal Process., 41 (4), 1532-1550, (1993). https://dx.doi.org/10.1109/78.212729

${ }^{29}$ Maragos P., Kaiser J. F., and Quatieri, T. F. Energy separation in signal modulations with application to speech analysis, IEEE Trans. Signal Process., 41 (10), 3024-3051, (1993). https://dx.doi.org/10.1109/78.277799 